\title{
Parasite-mediated interactions within the insect vector: Trypanosoma rangeli strategies
}

\author{
Eloi S Garcia ${ }^{1,2^{*}}$, Daniele P Castro ${ }^{1}$, Marcela B Figueiredo ${ }^{1,3}$ and Patrícia Azambuja ${ }^{1,2}$
}

\begin{abstract}
Trypanosoma rangeli is a protozoan that is non-pathogenic for humans and other mammals but causes pathology in the genus Rhodnius. T. rangeli and R. prolixus is an excellent model for studying the parasite-vector interaction, but its cycle in invertebrates remains unclear. The vector becomes infected on ingesting blood containing parasites, which subsequently develop in the gut, hemolymph and salivary glands producing short and large epimastigotes and metacyclic trypomastigotes, which are the infective forms. The importance of the T. rangeli cycle is the flagellate penetration into the gut cells and invasion of the salivary glands. The establishment of the parasite depends on the alteration of some vector defense mechanisms. Herein, we present our understanding of $T$. rangeli infection on the vector physiology, including gut and salivary gland invasions, hemolymph reactions and behavior alteration.
\end{abstract}

Keywords: Trypanosoma rangeli, Rhodnius prolixus, Parasite and vector interactions, Parasite development

\section{Review \\ Trypanosoma rangeli biological cycle}

The life cycle of $T$. rangeli, which it shares in invertebrate and vertebrate hosts, is complex and mediated by numerous factors, which are still poorly understood [1-4]. Infective parasites have been found mainly in the salivary glands of $R$. prolixus [1-4]; although $T$. rangeli has also been found in the salivary glands of Triatoma dimiculata in Colombia [5]. In the invertebrate host, the T. rangeli life cycle is characterized in three different regions of the insect vector: the gut, hemolymph and salivary glands, all of which are important for parasite development. The $T$. rangeli interactions in the vector begin with the ingestion of the trypomastigote forms in an infective bloodmeal. The parasites reach the gut of the insect vector and remain in the blood meal for some time after ingestion, but later they transform into epimastigotes that are able to multiply, and then normally cross the intestinal epithelium by an intracellular route and reach the hemocoel [1-4]. Then $T$. rangeli continues multiplying freely in the hemolymph or within hemocytes [6,7], although T. rangeli

\footnotetext{
* Correspondence: egarcia@fiocruz.br

${ }^{1}$ Laboratório de Bioquímica e Fisiologia de Insetos, Instituto Oswaldo Cruz (Fiocruz), Rio de Janeiro, RJ, Brazil

${ }^{2}$ Instituto Nacional de Entomologia Molecular (INCT-EM, CNPq), Rio de Janeiro, RJ, Brazil

Full list of author information is available at the end of the article
}

can be destroyed by plasmatocytes [7]. Thereafter, the flagellatesinvade the salivary glands, where they again multiply, and finally transform into metacyclic trypomastigotes, the forms that can be transmitted to mammalian hosts during a blood meal through salivary secretion [1-4,8-10].

\section{Hemocoel invasion}

In order to complete its developmental cycle in the insect vector, Trypanosoma rangeli needs to invade the hemocoel to overcome gut defense reactions [11-13]. Although it is known that $T$. rangeli decreases the growth of $R$. prolixus microbiota $[6,13]$, the mechanism that facilitates the survival and passage of the parasite from the lumen of the gut to the hemolymph needs further investigation. The midgut epithelium and possibly the gut perimicrovillar membrane of the vector gut represent fundamental steps in the life cycle of $T$. rangeli since they are related to the passage of the parasite from the midgut lumen to the hemocoel (Figure 1). Gamma irradiation causes changes in the ultrastructural organization of perimicrovillar membranes and microvilli in the gut, which leads to earlier parasite infection in the hemolymph in irradiated insects [14]. The ability of $T$. rangeli to attach to the gut surfaces of the insect vector is important for its development. Before invading epithelial tissues and/or cells epimastigotes have to find ways to attach to the gut lumen. Experiments 


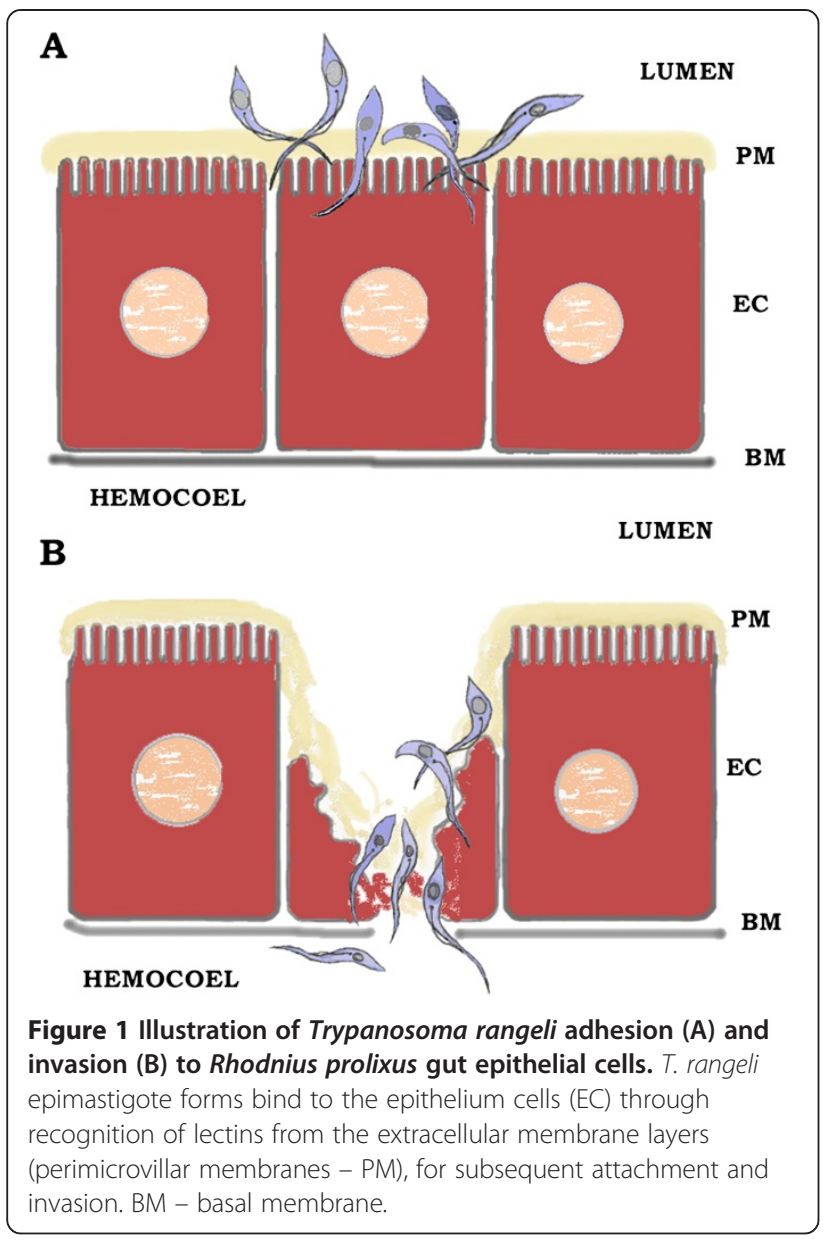

using scanning electron microscopy have demonstrated that $T$. rangeli attaches to the surface of some epithelial cells recognized by the parasites, for subsequent attachment and invasion. The parasites bind to the epithelium through the extracellular membrane layers (perimicrovillar membranes), and not to the plasma membrane layers $[14,15]$ (Figure 1). The presence of certain cells in the gut epithelium of $R$. prolixus is somehow recognized by the parasites for subsequent attachment and invasion [14,15]. In addition, some damage in the intestinal epithelium appears after parasite attachment [6,15] (Figure 1). However, other data have demonstrated that the penetration of $T$. rangeli into the gut depends on traversing the epithelial cells by an intracellular route without damaging the cells [8]. But in vivo and in vitro experiments have shown an association of several parasites with the same gut cell, and close contact between the parasites and the membrane layers. Also on cell penetration T. rangeli damages the surface, moving within the cytoplasm of the epithelial cell, and always in direct contact with the cytoplasmic organelles rather than the endocytic vacuoles (Figure 1). When the parasites reach the basal region, they cross the basal lamina, and enter the hemocoel $[6,8,15]$ (Figure 1). The success of $T$. rangeli to invade the insect hemocoel depends on both the parasite strain and the triatomine species. Usually, the insects are more susceptible to strains isolated from the same geographical region [16]. Even so, the invasion rate of the gut into the hemocoel is low, only about $10 \%$ of parasites pass through the gut cell wall. The remaining parasites present in the gut lumen are excreted with the digested blood meal $[1-4,8]$.

\section{Hemolymph interactions}

Although $R$. prolixus has an efficient system to eliminate pathogenic microorganisms, $T$. rangeli has the ability to survive in the hemolymph of $R$. prolixus counteracting the defense responses in many ways, and reaching the salivary glands to complete its life cycle in the invertebrate host [1-4,6,17-19]. In vivo and in vitro experiments have shown that oral infection with $T$. rangeli followed by inoculation of the insects with the same parasite inhibits hemocyte microaggregation reactions and release of arachidonic acid into the hemolymph of $R$. prolixus $[18,19]$. Additionally, a $T$. rangeli oral infection significantly reduces the phagocytic activities of $R$. prolixus hemocytes by inhibition of the PAF and eicosanoids pathways [20]. The mechanisms of this inhibition process is unknown, but some studies suggest that nitric oxide and superoxide could be the signaling molecules responsible to take the message from the gut to the hemocoel regulating anti-parasite reactions in this region $[21,22]$.

Once in the hemocoel, the parasite survives and multiplies freely on the hemolymph or penetrates into the hemocytes, especially plasmatocytes [6,7] (Figure 2). T. rangeli has been shown to overcome the hemolymph reactions, avoiding responses like, lysozyme and trypanolytic activity [23-26], prophenoloxidase (proPO) activation $[18,25-27]$, phagocytosis and hemocyte microaggregation $[18,19,28]$, hemolymph agglutination $[23,29,30]$ and superoxide/nitric oxide generation $[21,22]$. All these immune reactions are reduced by the parasite infection.

Some studies have revealed the presence of the short epimastigote forms of $T$. rangeli in the hemolymph during the first hours after hemocoel infection (Figure 2). After this period they transform into long epimastigote forms that are able to invade the hemocytes as well as the salivary glands [25,26] (Figure 2). The inoculation of the short epimastigote forms was able to activate the $R$. prolixus proPO system in the hemolymph while the long form was not [26]. This can be explained in part by the presence of a galactose-binding lectin purified from $R$. prolixus hemolymph that affects the survival and motility of short but not long $T$. rangeli epimastigotes forms [31].

Rhodnius species and/or $T$. rangeli strains may both be considered key factors for completing the parasite 


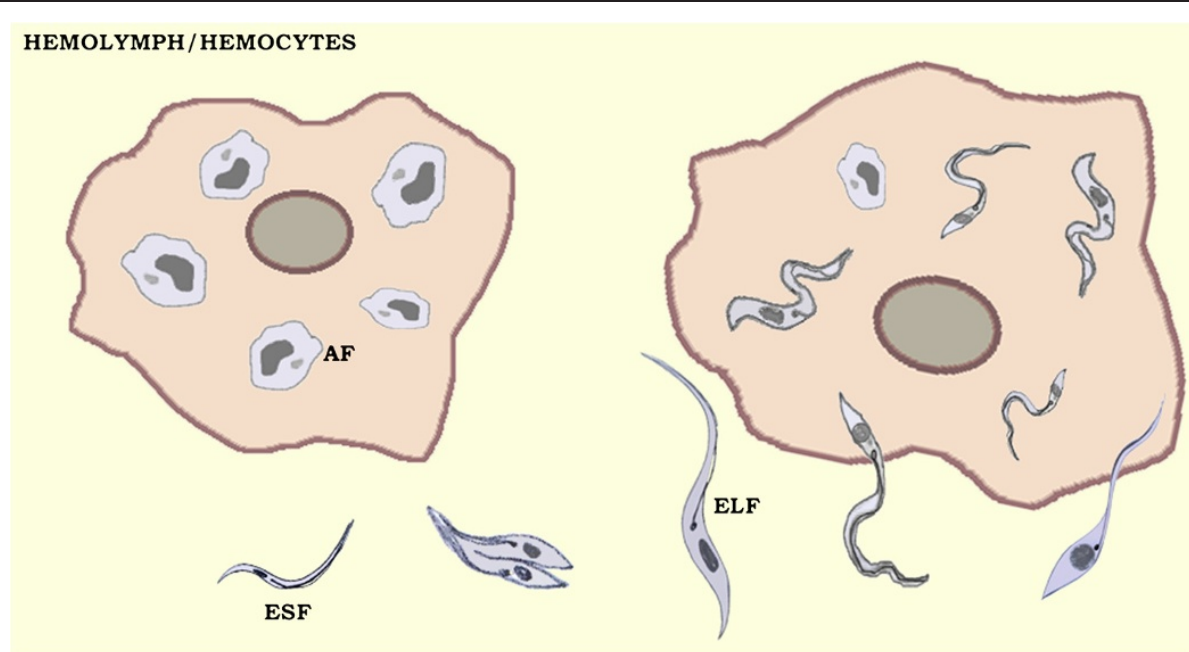

Figure 2 Illustration of Trypanosoma rangeli inside hemocytes and in the hemolymph of Rhodnius prolixus hemocoel. The parasite survives and multiplies freely in the hemolymph or penetrates into the hemocytes, especially plasmatocytes. T. rangeli epimastigote short forms (ESF) transform into amastigote forms (AF) or epimastigote long forms (ELF) which are able to invade the salivary gland.

development in the vector [32,33]. Recent investigations have demonstrated that the DNA mini satellites of the parasite may be involved in these interactions. The incubation of $T$. rangeli strains with $R$. prolixus hemolymph indicates the presence of a trypanolytic activity which acts against a $T$. rangeli $\mathrm{KP} 1$ - isolated from $R$. colombiensis, but has no lytic activity against the $T$. rangeli $\mathrm{KP} 1+$ strain from $R$. prolixus, both species were from Colombia. The survival of the latter strain suggests that hemolymph of $R$. prolixus seems to be a biological barrier which does not allow the development and transmission of KP1- [32,33].

\section{Invasion of salivary glands}

Detailed cytochemical characterization of Triatoma infestans and Panstrongylus megistus salivary gland cells have been described [34]. Invasion into the insect vector salivary glands by $T$. rangeli is necessary to complete its cycle and transmission to mammals, which is mediated by specific receptor-ligand interactions [35]. T. rangeli penetrates the $R$. prolixus salivary glands via the outer "membranes" disrupting the inner layers, in order to cross the basal lamina that surrounds the salivary glands and invades through the gland cells cytoplasm [35,36] (Figure 3 ). The parasite penetrates the flagellum foremost and then invaginates the gland cell to create a vacuole in which the trypanosome crosses the gland cells to reach the central lumen (Figure 3). After reaching the gland lumen, the epimastigotes remain adhered to the gland cell microvilli by their flagella, while metacyclic trypomastigotes are found swimming free in the saliva [35,36] (Figure 3). Epimastigotes cross the basal lamina through small holes to reach the glandular epithelium, which suggests that they produce a lytic molecule to allow them to pass through the epithelial barrier [35-37].
$R$. prolixus salivary glands are highly glycosylated and most protozoans have glycosylated compounds, as lectins or lectin-like molecules and enzymes, to regulate the parasite adhesion or invasion to host cells [38]. Since epimastigoteforms invade salivary glands, ecto-phosphatase activity of long epimastigote forms could be involved at the interaction sites of parasites and salivary glands with D-galactose and specific lectin-receptors [39]. Knowledge about salivary gland structures facilitates studies on the role of surface molecules in the attachment/invasion process by $T$. rangeli [38]. Rhodnius salivary glands have been shown to be rich in carbohydrate moieties on their surface, and present diverse lectin binding patterns with specific carbohydrate residues in the basal, muscle, and cell layers of the glands $[38,40]$. The carbohydrates detected on the salivary gland surface were used to investigate the adhesion between $T$. rangeli and the $R$. prolixus salivary glands. Experiments in vitro on attachment inhibition assays using long epimastigotes (the invasion/adhesion forms) demonstrated that some carbohydrates used were capable of inhibiting the receptors on both the salivary glands and $T$. rangeli surfaces [40]. R. prolixus salivary glands have several lectins which present surface-related sugars, and diverse carbohydrate residues are present in the basal lamina, muscle, and cell layers of the gland. Incubation of Con A reacted intensely with the whole salivary glands and with basal membrane in particular, indicating high concentrations of mannosyl residues [38,40]. In vitro sugar inhibition assays have demonstrated that some sugars tested attached to the surface of $R$. prolixus salivary glands and/or $T$. rangeli and inhibited the adhesion of the long epimastigotes to the gland surface [40]. The attachment inhibition tests, using parasites or salivary glands pre-treated with sugar revealed that the highest inhibitory 

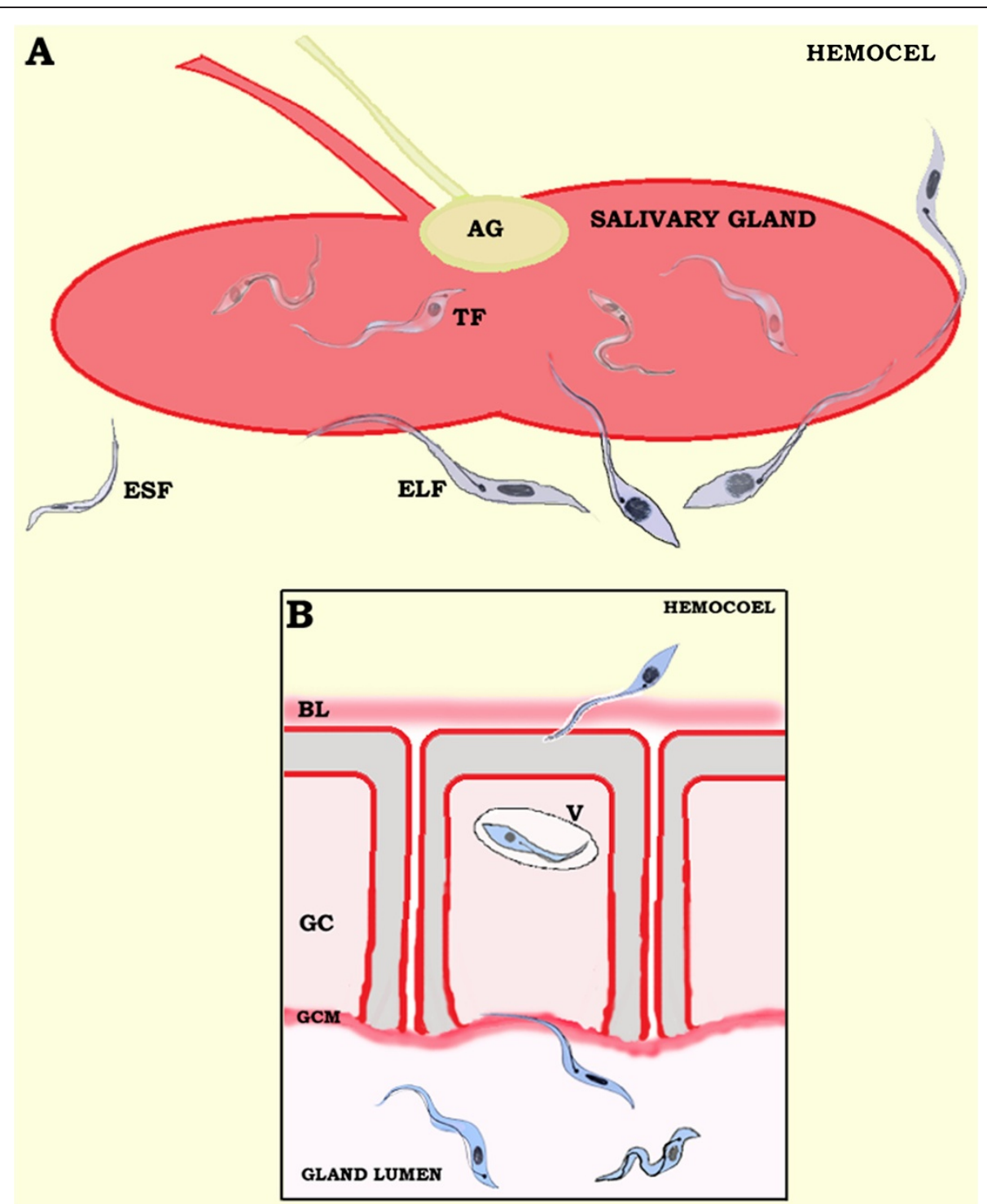

Figure 3 Illustration of Trypanosoma rangeli adhesion (A) and invasion (B) into the Rhodnius prolixus salivary gland. To cross the basal lamina (BL) that surrounds the salivary glands the parasite penetrates via the flagellum and invaginates in a vacuole $(V)$ in which the trypanosome crosses the gland cells (GC) to reach the central lumen. After reaching the gland lumen, the parasite epimastigote long forms (ELF) remain adhered to the gland cell microvilli (GCM) by their flagella, while metacyclic trypomastigote forms (TF) are found swimming freely in the saliva. AG - accessory gland; ESF - epimastigote short forms.

activities were observed with $\mathrm{N}$-acetyl-D-glucosamine, $\mathrm{N}$ acetyl-D-galactosamine, and galactose. These molecules may serve as receptors by which long forms of $T$. rangeli epimastigotes attach to the salivary gland surface, prior to invasion $[38,40]$.

\section{Saliva and alterations in behavior}

To overcome vertebrate reactions that prevent blood loss, saliva of $R$. prolixus contains dozens of different compounds with antihemostatic action, such as anticoagulants, antiplatelet and anti-inflammatory activities, and vasodilator compounds. Many of these biologically active salivary proteins belong to the lipocalin protein family [41-43]. The ultimate effect of this salivary antihemostatic apparatus is faster feeding by $R$. prolixus by decreasing the time required by the insect to locate the skin blood vessels and sucking blood efficiently $[9,44]$. The efficiency of salivary parasite transmission is increased by prolonged intradermal probing time on the host by infected insect vectors. $R$. prolixus infected with $T$. rangeli displays enhanced probing time, and that infection of the salivary glands affected the feeding behavior of the vectors increasing the number of intradermal piercings on a rabbit host reducing the ability to suck 
the blood when the insect is infected with the flagellate $[9,45]$. The prolongation of the probing time and the reduction of blood ingested in infected insects were not correlated to either the health of the insects or a physical obstruction of the food channel by the parasites by demonstrating that infected bugs probed and fed normally on a membrane artificial feeder [9]. Thus, it is probable that a $T$. rangeli infection causes salivary gland pathology that must contribute to transmission efficiency. In fact, saliva production was drastically reduced in insects with salivary glands infected with $T$. rangeli, as evaluated by plasma clotting time, apyrase activity, and NO-like compounds [9,41-43]. Since $T$. rangeli may damage the salivary gland cells of $R$. prolixus [8], salivary gland lesions could cause a deficiency in the biosynthesis processes of saliva components [9]. Thus, T. rangeli impairs $R$. prolixus salivary gland function, preventing full expression of its antihemostatic machinery. This led the insects to prolong the duration of intradermal probing, which favors $T$. rangeli transmission. Finally, $T$. rangeli infection in the hemolymph of $R$. prolixus leads to a delay in molting, alters insect movements and can increase mortality [46-48].

\section{Future perspectives}

There is ongoing research in our laboratory on the direct and indirect interactions between $T$. rangeli and $R$. prolixus in the insect gut, hemolymph and salivary glands, especially on the parasites cell tissue invasion. The flagellate mechanisms of the inhibition of cellular and humoral immune reactions in this insect vector need further investigation. However, it is becoming clear that some aspects of these gut and salivary gland invasions as well as the hemolymphatic compounds involved in this interaction are known. However, ligand-molecule interactions and several other surface molecules required for invasion of the gut and salivary glands must be better characterized. Additionally, epithelial immune reactions against the parasite invasion are poorly understood. Now, the increased availability of Rhodnius and $T$. rangeli functional genome analyses in combination with new experimental models, including doublestranded RNA knockdown, RNAi screens, transcriptomic and proteomic approaches, transgenesis, paratransgenesis of the vector and/or parasite, will offer a powerful tool for elucidating these parasite - vector interactions. Understanding how parasites are recognized as non-self, and how they have developed in the vector and the transmission strategies, facilitates the description of the molecular parasite-vector interface, vector competency, and provides unique opportunities to investigate the role of $T$. rangeli in shaping $R$. prolixus reactions against the parasite.

\section{Competing interest}

The authors declare that they have no competing interests.

\section{Acknowledgments}

This work was supported by grants from Conselho de Desenvolvimento Científico e Tecnológico (CNPq) to ESG and PA; Fundação Oswaldo Cruz (FIOCRUZ)/Papes project to PA; Fundação Carlos Chagas Filho de Amparo a Pesquisa do Estado do Rio de Janeiro (FAPERJ) to PA, and Instituto Nacional de Ciência e Tecnologia em Entomologia Molecular (INCT-EM-CNPq). ESG and PA are Senior Scientists from CNPq; DPC and MBF are Pos-doc

Fellowships from Coordenação de Aperfeiçoamento de Pessoal de Nível Superior (CAPES) and CNPq, respectively.

\section{Author details}

${ }^{1}$ Laboratório de Bioquímica e Fisiologia de Insetos, Instituto Oswaldo Cruz (Fiocruz), Rio de Janeiro, RJ, Brazil. ${ }^{2}$ Instituto Nacional de Entomologia Molecular (INCT-EM, CNPq), Rio de Janeiro, RJ, Brazil. ${ }^{3}$ Departamento de Química, Instituto de Ciências Exatas, Universidade Federal Rural do Rio de Janeiro, Rio de Janeiro, RJ, Brazil.

\section{Authors' contributions}

ESG, DCP, MBF, and PA conceived the review design, wrote the drafts and approved the final manuscript.

Received: 27 February 2012 Accepted: 30 May 2012

Published: 30 May 2012

\section{References}

1. Azambuja P, Garcia ES: Trypanosoma rangeli interactions within the vector Rhodnius prolixus - a mini review. Mem Inst Oswaldo Cruz 2005, 100:567-572.

2. Hoare CC: Trypanosomes of Mammals, A Zoological Monograph. Oxford, Edinburgh: Blackwell; 1972

3. Guhl F, Vallejo GA: Trypanosoma (Herpetosoma) rangeli Tejera 1920: an updated review. Mem Inst Oswaldo Cruz 2003, 98:435-442.

4. D'Alessandro A: Biology of Trypanosoma (Herpetosoma) rangeli Tejera. In Biology of Kinetoplastida, vol. 1. Edited by Lamsden VHE, Evans DA. London: Academic; 1976:327-493

5. Marinkelle CJ: Triatoma dimidiata capitata, a natural vector of Trypanosoma rangeli in Colombia. Rev Biol Trop 1968, 15:203-205

6. Watkins R: Histology of Rhodnius prolixus infected with Trypanosoma rangeli. J Invertebr Pathol 1971, 17:59-66.

7. Oliveira MA, de Souza W: Further morphological studies on the behavior of Trypanosoma rangeli in the hemocytes of Rhodnius prolixus. Parasitol Int 2003, 52:299-307.

8. Hecker $\mathrm{H}$, Schwarzenbach M, Rudin W: Development and interactions of Trypanosoma rangeli in and with the reduviid bug, Rhodnius prolixus. Parasitol Res 1990, 76:311-318.

9. Garcia ES, Mello CB, Azambuja P, Ribeiro JMC: Rhodnius prolixus salivary anti-hemostatic components decrease with Trypanosoma rangeli infection. Exp Parasitol 1994, 78:287-293.

10. Ferreira LL, Lorenzo MG, Elliot SL, Guarniere AA: A standardizable protoco for infection of Rhodnius prolixus with Trypanosoma rangeli, which mimics natural infections and reveals physiological effects of infection upon the insect. J Invertebr Pathol 2010, 105:91-97.

11. Castro DP, Moraes C, Garcia ES, Azambuja P: Inhibitory effects of D-mannose on trypanosomatid lysis induced by Serratia marcescens. Exp Parasitol 2007, 115:200-204.

12. Castro DP, Seabra S, Garcia ES, de Souza W, Azambuja P: Trypanosoma cruzi: ultrastructural studies of adhesion, lysis and biofilm formation by Serratia marcescens. Exp Parasitol 2007, 117:201-207.

13. Eicher S, Schaub GA: Development of symbionts in triatomine bugs and the effects of infections with trypanososmatids. Exp Parasitol 2002, 100:17-27.

14. Gomes AS, Graciano G, Nogueira NFS, de Souza W, Garcia ES, Azambuja P: Effects of gamma irradiation on the development of Trypanosoma rangeli in the vector Rhodnius prolixus. J Invertebr Pathol 2002, 79:86-92.

15. Oliveira MA, de Souza W: An electron microscopic study of penetration by Trypanosoma rangeli into mid-gut cells of Rhodnius prolixus. J Invertebr Pathol 2001, 77:22-26.

16. Machado PE, Eger-Mangrich I, Rosa G, Koerich LB, Grisard EC, Steindel M: Differential susceptibility of triatomines of the genus Rhodnius to 
Trypanosoma rangeli strains from different geographical origins. Int J Parasitol 2001, 31:632-634.

17. Garcia ES, Castro DP, Figueiredo MB, Genta FA, Azambuja P: Trypanosoma rangeli: a new perspective for studying the modulation of immune reactions of Rhodnius prolixus. Parasit Vectors 2009, 17:33.

18. Garcia ES, Machado EMM, Azambuja P: Effects of eicosanoids biosynthesis inhibitors on the prophenoloxidase-activating system and microaggregation reactions in the hemolymph of Rhodnius prolixus infected with Trypanosoma rangeli. J Insect Physiol 2004, 50:157-165.

19. Garcia ES, Machado EMM, Azambuja P: Inhibition of hemocyte microaggregation reactions in Rhodnius prolixus larvae orally infected with Trypanosoma rangeli. Exp Parasitol 2004, 107:31-38.

20. Figueiredo MB, Genta FA, Garcia ES, Azambuja P: Lipid mediators and vector infection: Trypanosoma rangeli inhibits Rhodnius prolixus hemocytes phagocytosis by modulation of phospholipase A2 and PAFacetylhydrolase activities. J Insect Physiol 2008, 54:1528-1537.

21. Whitten MMA, Mello CB, Gomes SA, Nigam Y, Azambuja P, Garcia ES, Ratcliffe NA: Role of superoxide and reactive nitrogen intermediates in Rhodnius prolixus (Reduviidae)/Trypanosoma rangeli interactions. Exp Parasitol 2001, 98:44-57.

22. Whitten MMA, Sun F, Tew I, Schaub GA, Soukou C, Nappi A, Ratcliffe NA: Differential modulation of Rhodnius prolixus nitric oxide activities following challenge with nitric oxide activities following challenge with Trypanosoma rangeli, $T$. cruzi and bacterial wall components. Insect Biochem Mol Biol 2007, 37:440-452.

23. Gregorio $E A$, Ratcliffe NA: The phenoloxidase system and in vitro interaction of Trypanosoma rangeli with Rhodnius prolixus and Triatoma infestans haemolymph. Parasite Immunol 1991, 13:551-564.

24. Gregorio EA, Ratcliffe NA: The distribution of agglutinins and lytic activity against Trypanosoma rangeli and erythrocytes in Rhodnius prolixus and Triatoma infestans tissue extracts and haemolymph. Mem Inst Oswaldo Cruz 1991, 86:181-186.

25. Mello CB, Garcia ES, Ratcliffe NA, Azambuja P: Trypanosoma cruzi and Trypanosoma rangeli: interplay with hemolymph components of Rhodnius prolixus. J Invertebr Pathol 1995, 65:261-268.

26. Gomes SAO, Feder D, Thomas NE, Garcia ES, Azambuja P: Rhodnius prolixus infected with Trypanosoma rangeli: in vivo and in vitro experiments. Invertebr Pathol 1999, 73:289-293.

27. Gomes SAO, Feder D, Garcia ES, Azambuja P: Suppression of the propheloxidase system in Rhodnius prolixus orally infected with Trypanosoma rangeli. J Insect Physiol 2003, 49:829-837.

28. Takle GB: Studies on the cellular immune response of insects toward the insect pathogen Trypanosoma rangeli. J Invertebr Pathol 1988, 51:64-72.

29. Ratcliffe NA, Nigan Y, Mello CB, Garcia ES, Azambuja P: Trypanosoma cruzi and erythrocyte agglutinins: a comparative study of occurrence and properties in the gut and hemolymph of Rhodnius prolixus. Exp Parasitol 1996, 83:83-93.

30. Mello CB, Azambuja P, Garcia ES, Ratcliffe NA: Differential in vitro and in vivo behavior of three strains of Trypanosoma cruzi in the gut and hemolymph of Rhodnius prolixus. Exp Parasitol 1996, 82:112-121.

31. Mello CB, Nigam Y, Garcia ES, Azambuja P, Newton RP, Ratcliffe NA: Studies on a haemolymph lectin isolated from Rhodnius prolixus and its interaction with Trypanosoma rangeli. Exp Parasitol 1999, 91:289-296.

32. Vallejo GA, Guhl F, Carranza JC, Triana O, Péres G, Ortiz PA, Marín DH, Villa $M$, Suáres J, Sánches LP, Pulido X, Rodrígues LB, Lozano LE, Urrea DA, Rivera FA, Cuba CC, Clavijo JA: Interacción tripanosoma-vector-vertebrado y su relación con la sistemática y la epidemiologia de la tripanosomiasis americana. Biomedica 2007, 27:110-118.

33. Pulido XC, Pérez G, Vallejo GA: Preliminary characterization of a Rhodnius prolixus hemolymph trypanolytic protein, this being a determinant of Trypanosoma rangeli $\mathrm{KP} 1(+)$ and $\mathrm{KP} 1(-)$ subpopulations vectorial ability. Mem Inst Oswaldo Cruz 2008, 103:172-179.

34. Anhê AC, Azeredo-Oliveira MT: Cytochemical characterization of Triatoma infestans and Panstrongylus megistus salivary gland cells (Hemiptera, Reduviidae, Triatominae). Micron 2008, 39:1126-1133.

35. Stark KR, James AJ: The salivary glands of disease vector. In The Biology of Disease Vector. Edited by Beaty BJ, Marguardt WC. New York: University Press of Colorado; 2008:333-347.

36. Ellis DS, Evans DA, Stamford S: The penetration of the salivary glands of Rhodnius prolixus by Trypanosoma rangeli. Z Parasitk 1980, 62:75-84.
37. Meirelles RM, Henriques-Pons A, Soares MJ, Steindel M: Penetration of the salivary glands of Rhodnius domesticus Neiva \& Pinto, 1923 (Hemiptera: Reduviidae) by Trypanosoma rangeli Tejera, 1920 (Protozoa: Kinetoplastida). Parasitol Res 2005, 97:259-269.

38. Ferguson MA: The surface glycoconjugates of trypanosomatids parasites. Phil. Trans. Royal Soc. London, Series B 1997, 352:1295-1302.

39. Gomes SA, de Souza AL Fonseca, Kiffer-Moreira T, Dick CF, Dos Santos AL, Meyer-Fernandes JR: Ecto-phosphatase activity on the external surface of Rhodnius prolixus salivary glands: modulation by carbohydrates and Trypanosoma rangeli. Acta Trop 2008, 106:137-142.

40. Basseri IF, Tew IF, Ratcliffe NA: Identification and distribution of carbohydrate moieties on the salivary glands of Rhodnius prolixus and their possible involvement in attachment/invasion by Trypanosoma rangeli. Exp Parasitol 2002, 100:116-234.

41. Ribeiro JMC: Role of saliva in blood-feeding by arthropods. Ann Rev Entomol 1987, 32:463-478.

42. Ribeiro JMC: Vector saliva and its role in parasite transmission. Exp Parasitol 1989, 69:104-106.

43. Ribeiro JMC, Andersen J, Silva-Neto MAC, Pham VM, Garfield MK, Velenzuela JG: Exploring the sialome of the blood-sucking bug Rhodnius prolixus. Insect Biochem Mol Biol 2004, 34:61-79.

44. Ribeiro JMC, Garcia ES: The salivary and crop apyrase activity in Rhodnius prolixus. J Insect Physiol 1980, 26:303-307.

45. Anez N, East JS: Studies in Trypanosoma rangeli Tejera, 1920.ll. Its effects on the feeding behavior in triatomine bugs. Acta Trop 1984, 41:93-95.

46. D’Alessandro A, Saravia NG: Trypanosoma rangeli. In Parasitic protozoa. Edited by Kreir JP, Baker J. London: Academic; 1992:1-54.

47. Schaub GA: The effects of trypanosomatids on insects. Adv Parasitol 1992, 31:255-319.

48. Schaub GA: Pathogenicity of trypanosomatids on insects. Parasitol Today 1994, 10:463-468.

doi:10.1186/1756-3305-5-105

Cite this article as: Garcia et al.: Parasite-mediated interactions within the insect vector: Trypanosoma rangeli strategies. Parasites \& Vectors 2012 5:105.

\section{Submit your next manuscript to BioMed Central and take full advantage of:}

- Convenient online submission

- Thorough peer review

- No space constraints or color figure charges

- Immediate publication on acceptance

- Inclusion in PubMed, CAS, Scopus and Google Scholar

- Research which is freely available for redistribution 